\title{
Formação de Redes de Consultoria Organizacional: o Lugar Especial dos Fatores Relacionais
}

\author{
The Making of Organizational Consultancy Networks: theSpecial Place of Relational \\ Factors
}

\author{
Laura Braga ${ }^{1}$ \\ Pedro L incoln C. L. de Mat tos 2 \\ Bruno Campello de Souza, D.Sc. ${ }^{3}$
}

\begin{abstract}
Resumo
As consultorias organizacionais têm-se mult iplicado e influem cada vez mais o dia-a-dia das organiz açõe s. Em alguns mercados há amplos espaços para médias e pequenas empresas prestadoras desse serviço. Cont udo, crescerte necessidade de e spe cialização, que tor na as compet ências das consultorias restr itas e suas estrutur as enxutas, tem est imulado a for mação de redes em que os consultores as completam e art c ulam. Par a est udar esse campo, cujas peculiaridades ainda são desconhec idas, est e art igo toma como refer ência a te cria de re des sciais, especialmente os fat ores de sua formação. Proc urou-se o relato dos próprios profis sionais sobre eles, prime ir o em um gr upo de noventa deles, part icipant es de um survey on-line. Em seguida, os r esult ados for am coment ados e m pr ofundi dade por um grupo deles, mais experiente, reunidos. Enqu anto os result ados se alinham, em geral, com os fat ores presentes em re des de plena imersão social (GRANO VETTER 1973; 1985), dest acam-se nesse caso a confiança pe ssoal no out ro consultor onde não se separa a com pet ênc ia técnica - e na e mpres a ass ociada, e a expectativa de comport amento ético. Elas se traduze mem regras, mesmo não esc ritas, e suportam os demais fator es de for mação e sust ent ação da rede.

Paburas Chave: Rede de Consultoria Organizacional. Confiança em con sultoria. Compro metimento em redes sociais. Co oper ação entrec onsu ltores. Ética em consultoria organizac ion al.
\end{abstract}

\section{Abstr act}

Or ganizational consultancies has been spreading and in fuencing more and mor et he ev ery-day life ofor ganizations. In some markets, enough room can be found for lit tle and middle sized firms providing this service. Nevertheless, the inc reasing need for specialization, whic h narr ows competencies and structures, st imulat es the formation of networks where consultants supplement and articulate themselves. In order to study this field, whose particularities are st ill unknown, the present art ic let akes as refere nce the theory of social networks, mainly the fact ors of heir format ion. These fac tor s were sought int he report of profes sional consultants, fir st in an on-line survey ofninety of the $m$. Sec ondly, results we re comment ed in-dept $h$ by a group of experienced consultants. Generally, findings are aligned tomain factors of full social immersion net works (GRANOVETTER 1973; 1985). However, per sonal tr ust in the ot her consultant - ins epar able of technical compete nce - and in the associat e consultancy, as well as e thic behavior, st and out. They bec ome manifest in rules, though not writt en, and the y support ather fact ars offormat ion and m aintenance ofnet work.

Key-words: Network of Organzation Consultancy. Trust in consultancy. Commitment in social net works. Co operation bet ween consultant s. Ethics in organizat ion consu t ancy.

1 Mestre em A dminis traça ão pela Univ ersidade Federal de Pe mamb uc o - Univ ersid ade F ed eral de Pe mambuco - UFPE. Pe qq uisadora d o PRO PAD NFPE. Rua EmaniBra ga, 377, ap to 102, Mada ena,Rec fe PE-Bra il.C EP 5061 0-350.E-mail: b r gabrtto@y aho o.com.br

2 PhD.P rofe ssor Titu lar d a Univ ersid ade F ederal de Pernambuc o (UFPE). Endereç o: Av. Be mardo V.d e Melo 1 264, ap. 1802. Pied ad e. a boatão - Perna mbuco B ra sil - CE P: $54400-000$ - E-mail: plincoln@hotlink.com.br

${ }_{3}^{3}$ Doutor em Psicobgia Cognitiva pelo Programa de Pós-G ra duaça o em Psicobgia Cognitiva da Unive ssidade Federa I de Perna mbuco -Professor Adjunto Depa rta mento de Ciênc ias Admini strativa s- Programa de PósGrad uação e m Ad min istra çã o - Un iversid ade F ed eral de Pernambuc o. Endereç o: Rua Gervá sio C ampe lon 102,Prado,Rec ife,Perna mbuc o-Bra sil-CEP50720-180 - E-mail:bca mpelo@uolcombr

A rtigo sub mtido em novem bro de 2007 e ac eitoem dez embrod e 2008 


\section{Campo, i nter esse e objetivos}

As consultorias organiza cionais surgiram na In glaterra, na pass agem do século XIX para o XX, em razão da necessidade de as org ani zações empre garem conh eci mentos de experts, que passaram a não mais atuar como funci onários dessas organizações, mas sim como agentes externos (DONADONE, 2001, 2003). Depois da Ingl aterra, foi a vez dos Estados Unidos e, as sim, as consul torias invadiramo mercado mundi al .

Ao se percorrer a hist ória da consultoria organizacional, conforme descrita por a uto res como Donadone (2001, 2003), v ei fica-se a ocorrên cia de alguns fenômenos típicos nas empres as dessa área de atuação. O primei ro deles é a vari abili dade de especiali zações, com modificação ou ampliação do foco de atuação segundo a demanda do mercado, sendo objetivo manter a consult ori a sempre apta a at ender a demanda dos se us cli ent es da forma mais co mpleta possív el. Outro fenômeno é o da miltinacionalização, desenvol vid ap elas consultorias a meri canas desde o início do século XX, que chegou a predominar no ramo. O terceiro fenômeno, vivido pel as grandes empresas de consultoria em todo o mundo, particularment e nos anos 80, 6 i o das fusões e aquisições qu etornou o setor uma nova potência ec onômica.

As grandes consult orias, as quais contam com elevado quadro de consultores, encontram-se at ual mente presentes no mercado brasil eiro (DONADONE, 2001, 2003), mas, n este c enário, os En ômenos ant eriormente apontados ocorrem de frma peculiar e paradoxal. De um lado, encontram-se as grandes consultorias mul tinacionais, que contam comestruturas gigantescas para atend er as demandas dos s eus clientes emdiversas e specialidades e até de forma comp let a (WOOD, 2004). Por outro, persist e a notória presença das pequenas e médias consultorias com necessidade de concentração em det ermi nadas especialidades que constituem o conjunto de co mp etências possuídas pelo pequeno grupo dos seus consult ores (MATTOS, 2005b).

Di ant ed eu mquadro onde o mercado continua a demandar diversida de crescente de servi ços de consul to ria, as pe quenas e médias consultori as, p or suas restrições de compet ênci a específi ca, encont ram-se incapacitadas de, individualmente, atender às exigênci as dos seus clientes, e pressi onadas por estes, vêem no arranjo de redes uma saí da, o que faz surgir, então, as redes de cons ultoria organi zacional. Trat a-se de arranjos colaborativos onde umgrupo de consultores e empresas de cons ultoria com compet ênci as especí fi cas atuam conj unt amente, passando a int er êrir, positiv a ou n egativa mente, na gestão da organização contratante.

Mizruchi (2006) chama a at enção para o fato de que a estrut uração de organi zaç̃̃ es em rede temsua explic ação em divers as perspectivas teóricas, entre as quais se encontrama ps iquiat nia (MORENO, 1934), a antropologia (BARNES, 1954) e a sociologia (SIMMEL, 1969). Cândido e Abreu (2004) acrescenta mà li sta a persp ectiva e conômi co-soci ológica (GRANOVETTER, 1985), a visão econômica (WILLIAMSON, 1975) e a Nova Economia Institucional (COASE, 1937). Os trabalhos de Campello de Souza (2004) e Pei xoto (2006) introduzem uma dimensão psicológica para o fenômeno. Assim sendo, tal tipo de arranjo não constitui fenô men o recente ou não teorizado (CÂNDIDO; ABREU, 2004), embora a apli cação dess a conceituação ao conte xto das consultorias or ganizacionais sej a ainda ine xplorada ${ }^{1}$.

Há uma escassez de estudos sobre red es emconsulto ri a o rganizacional, sendo a simples falta de registros nos sites de busca, mes mo trat ando-se de set or de serviço em grande evidência no cenário mundial, um bom indicado $\mathrm{r}$ disso. To ma-se nec ess ária, então, par a o entendi mento dessa indúst ria, uma investigação dos fatores associados à formação das redes, com uma ên fas e nos fato res sociais informais no sentido da "imersão s oci al" a rgumentad a p or Granovetter $(1973,1985)$.

\section{Conc eitos mai s pertinentes às redes em consult ori a}

\section{O conceito deredes}

Entende-s e por "red e" um conjunto de pessoas, grupos e/ou organizações que int eragem entre si através de contatos ativos e repetitivos que geram padrões de relacionamento relativamente estáveis (CARDOSO; ALVAREZ; CAULLIRAUX, 2002). Os partici pantes em questão podem ser ent endidos como "nós" cujas 
int erações ou fluxos de informação, recursos e/ou afeição constituem "traços" que os unem, fr mando a representação gráfi ca descrita por Barnes (1954 apud SÁ; ABELSON, 2004, p. 4) como "um conj unto de pontos, al guns dos quais unidos por linhas", um dia gra ma lógic o b ast ant e intuitivo (WELLMAN, 1982, 1988; CARVALHO, 2002). Tal estrutura int erconectada constit ui uma estrutura aberta, com capacidade para se expandir de forma ilimit ada pela integ ração de novos nós, bastando que compartilhemos mes mos códigos de comunic ação e efet iva mente se comuniquem(CASTELLS, 1999).

Dependendo da sua forma de constituição, as redes podem ser construídas a parti $r$ de rel ações int erpessoais, int erorganizacionais, int ergovernamentai s ou mes mo intersetori ais (GRANOVETTER, 1983; INOJOSA, 1999). Trat a-s e de ar ranjos so cialmente constituídos para suprir necessidades econô mi cas, políticas, so ciais e/ou cult urais dos s eus membros por meio de uma di nâmica desinergia emque o todo da rede émaior do que a s oma das suas rel ações (WELLMAN, 1988). Os nós qu e conectam os pontos de uma red epodem ser diretos ou indiretos (WELLMAN, 198 8), fortes ou fracos (GRANOVETTER, 1973, 1985), mas todos importantes em si mes mos ecomo $p$ art e do amplo sist ema social emque s eint egram.

\section{El ementos ess enciais às redes}

\section{Confiança}

A confiança é exercida por pessoas que agem em prol de outras pessoas, instituiçõ es e organi zações, na expectativa de serem recompensadas em algum pont o do futuro (KRAMER, 1999; BACHMANN, 2001). A expectativa de que o comportamento fut uro desejado e esp erado seja realmente o empregado pelo at or em qu ems e confiou (LUHMANN,2005).

A defini ção de con f ança carre ga três característi cas pri nd pais: vulnerabilidade, reciproci dade e expect ativa (PASSUELLO, 2 006). A vul nera bilida de está rel acionad a ao indivíduo que confia, o qual, ao decidi r confiar, está automaticamente incorrendo em ris cos, por não saber exatamente como o outro agi rá - característica reforçada por Luhmann (2005). A reci procidade corresponde à influ ência $d$ as interações cumul ativas no aumento ou na redução da confiança entre at ores int erdependent es - esta reforçada por Granovetter (1985) e Kramer (1999). Este último acrescenta que essa infl uência mút ua baseia-se em in frmação a respeit o de int enções, di sposições e motivos do outro que permitem a possibilidade de comportamentos esperados ou oportunísticos. Na expectativa, há uma frte relação com a a ssu nção de risc o atrela da ao at o de confiar. Neste, mantém-se a expectativa de qu eo outro aj a da frma esperada - caract erística reforçada pel os já mencion ados Kramer (1999) e Luhmann (2005), além de Bachmann (2001) e Lane e Bachmann (1996) -, e se reduz, s egundo Gulati (1995), à a preensão sobre o agir oportunístico. É sob esta expectativa que se estabelece a disposição para ass umir risco (LUHMANN, 2005).

Assim, a confiança é um mecanismo de cont rol e soci al (LANE; BACHMANN, 1996; BACHMANN, 2001; GULATI, 1995) que reduz a incerteza e a compl exi dade das relaçõ es sociais, a o mes mo tempo em que produz risco para aqu ele que confa (LUHMANN, 2005). O risco de confiar pode ser muito el evad o (BALESTRO, 2002), e cabe a cada i ndivíduo, em cada relação, decidir sobre a viabilidade de confi ar no outro. Quanto mais bo as razões para acreditar que o outro é digno de confi ança, maiores as chances de se assumi r o risco da conf ança. O inverso t ambém ocorre quan do há motivos para se acreditar que o outro não é digno de confiança, o que ol eva a se abster de ass umir o risco de confiar (LUHMANN, 2005).

A f mde to mar uma deci são mai s acertada quanto a confiar ou não, os atores sociais buscam informações sobre os outros (PUTNAM, 1996). Quanto mais s eguras frem estas informações, mais provável será que o ator as leve em consideração emsua t omada de decisão - inclusive pelo fato de ser em limitadas (GRANOVETTER, 1985), pass ando a constitui $r$ boas razões para conf ar ou não (LUHMANN, 2005). Informações advindas das mais divers as fontes são úteis e necessárias - a principal refere-se ao históni co das relações entre os próprios a tores envolvidos natomada de decisão - o" confiante" e o "confiado". 
Com base em diversos elementos fornecedores de informações sobre confar ou não, al gumas for mas de conf ança são disti nguidas por di versos autores como Lane e Bachmann (1996), Luh mann (2005) e Bal estro (2002).

No cont ext o organizaci onal, Passuello e Swirski de Souza (200 5) trabalham com três formas de con fança: i) conf ança a parti $r$ das caract erísticas pessoai s dos indivíduos envolvidos nas interações - os consultores -, desenvolvida no compartilhamento de experiências e conhecimento entre eles; ii) confiança que se vale da expertise do out ro - o consultor parceiro -, que pode funcionar como suporte em determinadas áreas específicas, est ando os indi víduos ligados não ma is por características pessoais, mas por compet ênci as específicas; iii) confiança com base nas características da org ani zação, calcada, segundo Gulati (1995), na reput ação d a i nstitui ção - empre sa de consultoria ou consul tor autônomo -, ou n a forma co mo as questões são conduzidas e os problemas resolvidos por ela; bas eia-s e em padrões e normas, formais ou não, e no desempenho.

Rennó (2001) af rma que a confiança interpessoal (basead a emcaracterísti cas pessoais e na expertis e do outro, nos diri gentes ou representantes das instit uições/organizações) é condição essencial para a con fiança nas institui ções (systemt rust).

\section{Compromet imento}

Nas pala vras de Anders on e Weitz (1992, p. 19): “[...] comprometimento com uma relação requer um des ejo de desenvolver uma rel ação estável, uma vontade de fazer sa cri ficios de curto prazo para manter a relação, e u ma s egur ança na estabilidade da rela ção".

St anl ey e Markman (1992) afirma mque o comprometi mento abrang e dois const nut os relacionados: dedicação pessoal - investi ment o na relação, co m busca, não apenas do próprio bem estar, mas do relativo a todos os membros nel a envolvi dos -; e comprometimento cerceado (constraint commitment) -, constrangi mento sobre cada membro para permanecer na relação, independ entemente de sua dedicação pessoal, tratando-se, port anto, de uma garantia para o comprometimento. De acordo com autores como Anderson e Weitz (1992), Stanley e Markman (1992), Mo rgan e Hunt (1994) e Al meida, Co elh o e Coel ho (2004), al guns elementos funcionam diretamente como cerceadores do comprometi mento com a rel ação, como: investimento anterior na relação, c omunic ação (de mão dupla aberta), valores compartilhados, be neficios da relação, p ressão social, desgaste de procedimen to de término, $\mathrm{n}$ ão atrativi dade das alt ernativas, indis poni bilidade de parceiros e a dependência já consolidada entre eles. A con frança, ela própria, é elemento precursor da rel ação (ALMEIDA; COELHO; COELHO, 2004), mas de frma indireta (MORGAN; HUNT, 1994). De acordo com Almei da, Coel ho e Coelho (2004), ela só teme feit o positivo sobre o comprometi mento qu ando se encont ra emníveis elevados.

Assim como elementos que garantemo comprometi mento, existe também o que o compromete, ou sej a, qu e é consider ado destrutivo para o comprometimento dos atores emsuas relações: a históri a d e conflitos envolvendo as partes ou al go a elas relacionado.

Os resultados do comprometimento são: redução da propensão a abandonar (a relação), aumento do consenti mento e crescente cooper ação. Se os atores estão co mp rometidos é porque acreditam que a rel ação é i mport ant e e valiosa e, portanto, deve perdurar. Lo go, em havendo comprometiment o, reduz-se a p ropensão dos atores - de acordo como modelo de Anderson e Weit z (1992) - a deixar a relação. O cons entimento e a cooperação são distinto s. O primeiro é reativo, ao passo que o segundo é proati vo. Ao cons entir, o ator aceita passivamente as decisões do outro. Ao cooperar o ator interag ep roativamente coms eu par, em busca da mel hor forma de alcançar objetivos mútuos. A parte comprometida com a relação cooperará com o o utro membro porque deseja fazê-la funcionar (MORGAN; HUNT, 1994).

Al émde t odos os elementos supracit ados, out ro está di retament e ligado, de frma g eral, ao co mp rometimento: a percepção de comprometi mento da outra part e. Assim, A só s e comprometerá comB se perceber que B está comprometido consigo (A). Logo, o compromet iment o de um ator é causa e éfito do comprometimento do outro.A rel ação de comprometimen to é,p ort ant o, cí clica (ANDERSON; WEITZ, 1992). 
O ciclo que se forma da perc epção de A (sobre o comprometimento de B) até o comprometi mento de B (que perœbeu o comprometimento de A) gera um reforço positivo que, por sua vez, leva ao aumen to do comprometimento, no tempo, de ambas as part es. Contudo, se houver a percepção de uma das partes de que o comprometimento é assi métrico, ou seja, qu e um at or est á ma is comprometi do que o outro, t enderá a o correr uma ins atisfação com a relação. $O$ ator mais comprometido se torna mais vulnerável na relação; há u ma disposição maior do menos comprometido a romper co ma relação e, da parte mai s comprometida, uma menor disposição para reali zar s acrific ios .

\section{Cooper ação}

A confiança está cal cada na reciprocidade da relação, que, de ac ordo com Axel rod (1984) baseia-se na id éia de um jogo de "soma positiva", em que todos ganham e é possibilitado pela redução do risco decorrente da mi nimização dai nœert eza sobre o comportamento do out ro (LOPES; BALDI, 2005). Est a mesma reciprocidade está no âmago da cooperação para a busca de interesses e objetivos comuns, reduzindo os riscos de comport ament os oportunísti cos. Nest e jogo, cada jogador é tentado a s e comport ar de maneira oportunist a, mas o compo rta mento c ooperativo mútuo levará todos ao melhor des empenho (BALESTRO, 2002), base ando -se na consciência da i mport ância re cíproca dessa c ooper ação para os p articipa ntes (PUTNAM, 1996).

A mes ma lógica é expli cad a pela Teoria dos Jogos por meio do chamado "Equilíbrio de Nash", em que os indivíduos em competição pelo mesmo bene fi ci o resolvem coop erar umco mo outro para não correr o risco de um dos dois f car emprejuízo. Au seja, eml ugar de uma s oma zero, gerada pel a competição emque um ganha e o outro perde, gera-se a possibilidade de ganhos mútuos (PUTNAM, 1996), a s upracitada "soma positiva". A fil osofi a inerente é a do ganha-ganha, mantendo-se o foco na relação como um todo, e não em u ma única int era ção. Assim, cada ator pode até ganhar menos emuma det ermi nada transação relacional, mas os result ados da rede serão mai s benéfi cos do que a soma de i nterações isol adas e fora dela.

Como evidenciado acima, a confiança e a cooperação apresentam-se de forma conjunt a, sendo a confi ança tanto result ado como mei o da interação cooperativa. Assim, quanto mais elevado o nível de confi ança, maior a probabilidade de haver coop era ção, e vice-versa. Esta confian ça, por sua vez, dev e s er mútua, ou seja, não basta a con fiança no out ro; para cooperar é preciso ainda acreditar que se goza da confiança do outro (PUTNAM, 1996). À confiança deve ser adicionado o comprometimento, para que se desenvolva a cooperação (MORGAN; HUNT,1994).

As re des de cooperação entre firmas, i nicialmente independent es, result am, para tais empresas, em i mportantes vantagens no mercado, as quais seri ami mprová vei s de serem alcançadas por outra forma que não pela est rut ura em rede. Di f cul dades também existem. Problemas inerentes a esta forma estão presentes, mas em menor proporção e rel evância que as vantagens (BACHMANN, 2001). Os tão temerosos conflitos exist ent es nas rel ações não d esaparecem com o est abeleciment o de uma relação base ada em confiança, comprometi ment o e c ooperação. Eles continuama existir, pois são inerentes às relações. Cont udo, emu ma rel açã o basea da nos t rês elementos supracitados, os conflitos nos quais se incorres ão transformados em conflit os funcionais, ou seja, resolvi dos ami gavelment e, comvi st as ao al cance dos objetivos mút uos (MORGAN; HUNT, 1994).

\section{Método}

Como não há re gistro de conhecimento sistematizado e objetivado a res peito do fenômeno redes de consultoria organizacio nal ${ }^{1}$, este estudo pode ser caract erizado, segundo Cooper e Schindler (2003), como uma pesquisa explo rat ória. Seu foco está nos consult ores e empresas de consultoria organizacional de pequen oe médio portes da Região Met ropolitana do Recife, nas mais diversas especialidades e foco s d ecompet ênci a.

A coleta de dados sed eu emdu as fises: uma quantit ativa, outra qualitativa.

Fase quantitativa. Foi reali zada entre 18 de outubro de 2006 e 06 de novenb ro do mesmo ano, com a apli cação de um frmulário on-line para 90 consultores, que li vremente se associaram ao cadastro de 
correspondência de um grupo de pesqui sa sobre consultoria organizacional da Universidade Federal de Pernambuco. Eram obj etivos: a) traçar o perfil destes consultores, e b) identi f car, segundo a percepção dos próprios consultores, a influência de alguns fatores relacionais - segundo a teoria geral de redes, aci ma s umari zada - na formação de redes de consultori a organizaci onal. No formu lário eletrôni co, um conjunto de enunci ados descrevi a aqueles fatores supostamente influentes, e uma escala Lik ert propunha quat ro graus em que o respondente indic aria a precisão com que tal enunciado representava a realidade ("representa bem", "representa co m restrições", "represent a superfici al mente" e "não representa"). Foram medidos os seguintes fat ores na brmação das redes: a compl ementaridade como diferencial da rede; a expect ativa de acesso e manuten ção do mercado; a expect ativa de melhori a comum; o comprometi mento de um parceiro con frme comprometimento do out ro; as regras como redutores de risco; a consciên cia do risco; a reciprocidade da c ooperação; a expectativa de comport ament o éti co; a relação prévia (à formação da rede); o tamanho da rede conf rme o mercado (s ua necessi dade); a integração dos me nb ros pel a prática; as interações pessoais (extrarede); a natureza da rede descrita: se integrada por consultorias, se por consultores autôn omos ou se por ambos ("rede mista").

Fa se qualitati va. Consisti u na reali zação de uma entrevist a coletiva semi -estruturada, de três horas de du ração, cuida dosamente pl anejad a e preparada, com quatro cons ultores de perfis distintos (pertencentes a empresas de consultori a médias ou pequenas, de redes constituídas formal ou informalmente, e aut ônomos) no di a 23 de novembro de 2006. Embora s eguindo rotei ro previamente passado aos partici pantes, deu-s e liberdade para desenvolviment os espont âneos. O objetivo era buscar explicaçõ es qualitativas para os result ados quantit ativos (sur vey ant erior com 90 consultores), apresentados em tabel as aos quatro partici pant es, com destaque para a guns números, que representavamai mport ância maior ou menor de certos fitores na formação da rede.

A anális e quantitativa se deu pela apli cação de técnicas estatísticas descritivas sobre as questões de perfil do respondente e de id entificação dos fatores rel acionais, e i nf erenciai s (mat riz quadrada de co relações Spe arman Rho) sobre os fitores rel acionais e a relação destes com as questões de perfil. Os textos provenientes da entrevist a coletiva foram analisado s qualitati vamen te, em três etapas como sugerido por Mattos (2005a): i) recup era ção do mo mento da entrevista, o movi mento da conversação e su as inflexões, incluindo-se anotações fei tas durante ela; ii) análise do si gni f cado semântico e pragmático dos diálogos, com bas e nos pontos do roteiro da entrevist a e nos que emergiram da própria di nâmi ca da conversação; iii) validação posterior, pelos entrevist ados, dos trechos es colhidos para uso pel a pesquis adora.

\section{Resultados}

Para um ent endimento mais claro e holístico dos resultados, primeiramente serão apresentad os apenas os qu antit ativos, f cando os qu alitativos para a seção se guinte, já dent ro de uma discuss ão g eral dos resultados, inclusive porque estes representami nterpreta ção daquel es, pelos próprios cons ultores.

\section{Análise quant it ativa}

Os respond entes fram, em sua maioria, consultores homens (67\%), comidade na fiixa dos 46 aos 65 anos (67\%) e formação em ní vel de especi alização $(51 \%)$. As áreas em que at uavam eram pri ncipal ment e gest ão, estrat égi a, recursos humanos, plano de negócios, qualidade, marketing, est udo de viabilidade ec onômi ca e gest ão do conheci mento. Não houve, cont udo, exclusividade de uma área, ou seja, a grande maioria dos consultores presta consultoria em mais de uma áre a $(88,9 \%)$. Cerca de $80 \% \mathrm{t}$ inh amexperi ência de consultoria de, no míni mo, 05 anos e 37,5\%, mais de 15 anos ( $\mu=12,91$ anos). Esse perfl dos ent revistados confere-lhes maturidade pessoal e prof ssional para fornecer ampla visão do mercado pesquisado, incluindo-se s uas diversas áreas específicas.

A maior part e dos pesquisados (73\%) estava vi nculada a al guma empresa de consult oria: $27 \%$ trabalhavamde forma autônoma; $72 \%$ diret amente com, no máximo, 05 consult ores, tendo na consultoria sua atividade profissional principal. São representativos, portanto, do campo pesquisado, consul torias de pequeno e médio portes. 
Quanto à participação em rede, a amostra se apres ent a bem dividida: 53\% partici pam ou já participaram de a lguma rede de cons ultoria organizacional; 47\% nunca participaram Da queles que já haviamparti cipado, $14 \%$ consideraram a experiência "muito gratificante"; $57 \%$, "g ratificant e"; $27 \%$, "complicada"; e apenas 2\%, "frust rante" - tendo-se um total de $71 \%$ que julgam sua experi ência, no míni mo, gratificante. Sustentam, port anto, a grande relevânci a, atual id ade e tendência de cresciment o dess e arranjo organizacional no ca mpo da consultoria.

Dos fatores rel acionais medidos, os de maior força na frmação das redes foram:

_ "expect ativa de éti ca" ( $80 \%)$;

¿ “co mp lementaridade como difer enci al " (71\%);

- “integração pel a prática" (70\%);

- "co mp osi ção mis ta (empresas e autônomos) das redes" (67\%);

- "reciprocida de na coop eração" (64\%);

_ “expect ativa de mel horia comu m" (61\%);

—_"regras como fatores redutores de ris co" (60\%);

- "consciência do risco (57\%)";

- "expect ativa (quanto a) ac esso e man utenção do mercado" (57\%);

A frça dos percentuais aci ma demonstra a grande rep resent atividade dos fat ores rel acionais na frmação das redes de consultoria or gani zacion al. O relacionamento ético entre os membros da rede, adiante explicad o na investigação qual it ativa, dest aca-se de entrada. Confian ça, cooperação e comprometi mento, com percentuais equivalentes, podem ser apontados, seguindo a literatura, como o núcleo essencial da rede. Eles estão, respectivamente, na consciênci a do risco - inerent e ao at o de conf ar (57\%) -, na recip rocidade da cooperação, qu eé s eu âmago (64\%), e na expect ativa de mel horia comu m, objetivo do comprometimento $(61 \%)$.

Os demais fatores da formação de rede (comprometimento conforme co mprometimento, relação prévia, t amanho conforme mercado e int erações pesso ais) mostram a mesma tendência, mas com percepções menos consensuais no detal he dessa car act erísti ca relacional. (Tabela 1, apêndice A).

As interações ent re as vari áveis rela cionais da formação de redes de consultoria foramavali adas por inter médio de uma mat riz quadrada de correlação (co ef ci ent e Spear ma $n$ Rho). As correlações mai s elevadas apont am uma corr elação entre a inte gração pel a práti ca e a cooperação conforme co operação - correlação de 0,47 (vid etab ela de correlações, apêndice B). A independência das variáveis em rel ação às diferenças de perfil profission al dos component es da rede podei ndi car a forç a niveladora das rel ações est abelecidas pel a associa ção emre de.

\section{Di scussãogeral dos resultados}

\section{Formalização ou não das redes}

Os cons ultores entrevistados concordam que as redes podem ser formais. No ent anto, de acordo com o Consultor 4, "para uma rede funcionar eu não preciso frmali zar. [...] uma red e que não tem formalização e funci ona o tempo todo, independente de eu dar a el a um CNPJ" (ent revista coletiva, 23/11/2006). Assim, indep endentemente da frma como a rede venha a ser estabelecida, o que defi nirá sua existênci a s erão: sua int egração pel a p rópria prática, traduzida em cooperação i nterna; inclus ão das consultorias demandadas p elo mercado; perspectiva de lon go prazo; postura ética; comprometi mento mútuo; e est abelecimento de regras, em cumprimento ao seu objetiv o de indicaçõ es entre os membros para at ender à demanda global de seus cli entes (vide Tabela 1 no Apêndice A). E mais: troca de experiências, informações e geração de conhecimento, alcançando umdi erencial competitivo. 
De acordo com $83 \%$ dos consultores, regras e normas devem ser estabel ecidas no processo de fr mação das redes para reduzir os riscos e assegurar sua int egraçã o na prática, o que remete à literatura (JOSSERAND, 2006 apud LIMA; LIMA; TAKAKI, 2004). Para estes $83 \%$, es tas regras e normas são est abelecidas pelo grupo, sendo, portanto, de forma geral, coerentes com as crenças dos me nbros. Seu objetivo é regul ar o comportamento de todos os consultores e empresas de consultoria que se propuserem a atuar na rede, incorrendo na redução dos riscos rel ativos a comportamentos in desejados. Entre ess as regras destaca-se a ve dação à prática do "comissionamento" (um consultor paga a outro comi ssão p or ter sido po r est e indicado a um cliente), para evit ar a concorrência interna ent re os membros da rede, e a an uência à entrada de novos membros. Nas palavras do Consultor 3:

Não elimina a co ncorrência, mas ela tem que ser regulada, [.. .] deve se pautar por um có digo de éti ca. [...] E neste código de ética é absolutamente indispensável que os membros não recebam comissionamento. Isso é absurdo, é antiético. Outras coisas também não são permitidas, como por exemplo, [...] fazer prospeç̧ão em diente que já é atendi do [por outro consultor]. É absolutamente condenável. Isto tem que faz er parte do código de ética. [...] empresas que se propõem a atuar sob o guarda-chuva de uma marca [a da rede de consultoria], de um conjunto de princípi os, têm que ter um código de ética. (Consultor 3 , entrevista coletiva,23/11/2006)

As regras e normas podem ser estabelecidas brmalmente, com um código de ética discutido e aprovado por todos, ou ta citamen te, de a cordo como Consultor 3, ed e acor do como consul tor 4, emn egociações recor rentes entre os membros. Independentemente da forma pela qual sejam estabel ecidas as reg ras, os consul to res af rma m que devem ser cumpridas, sob pena de exclusão da rede.

O tamanho das redes é det ermi nado pelo mercado (existência de demanda e capaci dade de atendi mento), s egundo $72 \%$ dos consultores, mas seguramente limit ado pel a possibili dade real de integração entre seus membros.

É o mercado quem det ermina. [..] [Se não,] cria um dube, clube de convivência. Você tem que estar ali é para at ender a uma demanda, ent ão éo mercado que det ermina o tamanho. [Mas] vo cê não vai sacrificar, digamos, a convi vência e a própria experiência por causa do tamanho. Você tem uma reuniã o com 50 pessoas,já não dá nem para conversar. (Cons ultor 3, entr evi sta coleti va, 23/11/20 06)

\section{Níve is de int er ação}

Assim co mo os format os de rede de consultoria organizaci onal vari am, o mes mo ocorre com as interações int emas. Est as podem dar-se entre empresas de consultoria, entre consultores autôno mos/independentes ou lig ados a empresas de consultoria, ou até mes mo em u m misto destas duas formas. Em redes constituídas por consultores só há possibilidade de rel ação interpe sso al. Já naquelas constituídas por empresas de consulto ria, as int erações pod em ocorrer tanto no nív el pes soal quant o no organizacional, em que prevalec em, segundo $67 \%$ dos consultores, as de nível pessoal devi do à personi f cação da consult ori a na f gura do consultor. Esta situação é ev idenciada nas empresas com umúni co consul tor, como a firmado pelo Con sultor 4 :

[...] é um "p essoal”, mas que se r estringe [ao indi víduo] enquant o profissional. [..] el es [o pessoal e o organizacional] se confundem, até porque você vai ver que grande parte das empresas [referindo-se ao perfil da amostra quantitativa], tem menos de 5 empregados: o consultor e sua equipe de suport $e$. (Consultor 4, entrevista col etiva ,23/11/2006)

A grande i mport ância das interações pessoais nas redes de empres as de consultori a, que se sobressaem em rel ação às interorganizacionais no process o de formação, reside em dois el ementos: i) na personifi cação da consultori a na figura do consultor; e ii ) no fato de a decisão de se inseri r na rede calcar-se no con heci mento do outro e na confiança quanto à possibilidade de contribuição dele para a rede. É pertin entes alientar que as int erações pessoais se mantêm no ní vel profission al - $\mathrm{n}$ ão dizem respeit o a proximi dade ou amizade pessoal. Isso poderá s er verific ado logo adi ant e, ao se tratar da confian ça. 
Contudo, est abelecidas as redes, há si nais de que, naquelas constituídas por empresas de consulto ri a, ocorre t ambém um acréscimo de i mp ort ânci a das int erações organizacionais, interações entre empresas ou marcas, mas s emprejuízo d as interpessoai s, como diz o Consultor 3:

[...] eu diria que à medida que o tempo vai passando diminui op essoal e aumenta o or gani zacional, aliás, diminui não, mantém-se o pessoal e aumenta o organizacional, porque são empr esa sue apr endem a conviver como organizações. (Consultor 3, entrevista coletiva,23/11/2006)

\section{Confiança}

O est abeleciment o da conf ança entre os consultores e as empresas de consultori a é consi derado pel os quatro consultores entrevist ados essen cial para que poss ams e unir emuma red e - o que remete a Bachmann (2001) e Morg an e Hunt (1994). Assi m, os consult ores confiam em out ros consultores (independentes, autônomos ou pertencentes a uma empresa de consul toria) ou emempresas de consult ori a. Como as pri meiras interações são pesso ais - e há, in clusive, uma predominân cia dessas em relação às organizacionais -, a confiança gerada é inseparável da competência indivi dual (o consultor enquanto profissional), denomi nada por Passuello e Swirski de Souza (2005) como confiança a partir da expertise do outro. Nesse processo de formação das redes de consultori a organizacional, também ocorre o reverso: a confiança pel as caracterí stic as da organização, mas s ecundariamente, pois ela só se constitui após o estabel eci mento da confiança pessoal, como a f rmado por Rennó (2001). A pres ença dos dois tipos de confian ça ea ligação entre elas podems er percebidas no di scurso dos próprios consultores: "Si mples mente confo int egralmen te nas empresas deles e neles pessoalment e. São pesso as altamente competentes" (Cons ultor 4, entrevist a col etiva, 23/11/2006).

A expectativa de $93 \%$ dos consult ores éd eque cada um adote e manten ha uma postura éticaemt oda a história da rede -, esta expectativa é ab ase para o estabelecimento da confiança.

De acordo com $83 \%$ dos consultores, con far em outros consultores e/ou empresas de consultoria ac arret a a a ssun ção cons cient e de um risco, representa do pela possi bilidade de que aqueles em quem se confia aj am de forma diferent e da esperada, o que, aliás, é inerente ao ato de confi ar (KRAMER, 1999; LUHMANN, 2005; BACHMANN, 2001; LANE; BACHMANN, 1996).

Com ên fase maior que os números do survey, os consultores entrevistados apresentam, como p ré- requisit o $\mathrm{p}$ ara confar, a necessidade de relação prévia, geralmente profissional, entre os futuros membros da rede, fonte s egura e imprescindível de informações (GRANOVETTER, 1985), nas quais se basei a a expectativa de comport ament os éticos fit uros.

Rel ação entre os membr os, prévia à formação da rede, é necessária [...], sem dúvida, porque eu acho que o que faz com que as pessoas trabalhem ju ntas é a confiança. Par a que aconteça isso é preciso que vo cê tenha um conhecimento das pessoas. (Consultor 1 , entrevista coletiva, 23/11/2006)

Sem ter esse conhecimento prévio, sem eu confiar em quem eu vou trabalhar junto, quem eu vou colocar dentro do meu diente, não se começa uma rede. (Consultor 2, em entrevista coletiva, 23/11/2006)

De acordo com os consultores ent revistad os, a relação prévia pode dar-se por uma relação de trabalho anterior, em que os membros tenham atuado, concomit antemente, em um cliente ou mesmo em uma interação já direci onada à própria intenção de inserção na rede.

O ing resso na rede é ocasião emque a questão da confiança se revela.

Só pode entr ar [na rede] se houver vaga e for indicado por u m, e essa indi cação significa que a pe ssoa se submete a fazer uma apresentação de sua empresa. Tem uma defesa, a pessoa apres enta. A condição para entrar é [não ter] um veto. Eu não posso viver com alguém que eu não confito. (Consultor 3, em entrevist a coleti va, 23/11/2006) 
O est abeleciment o da confiança e a expect ativa de comportamentos éti cos, segu ndo $82 \%$ dos consultores, são indispensáveis para que haja coop eração ent re as partes, conforme o Co nsultor 3: "empresas que se propõema atuar s ob o guarda-chuva de uma marca, de um conjunto de princípios, têm queter um código de ética" (entrevista col etiva, 23/11/2006). Tal c ooper ação, co ntudo, não set raduz na retribuição de i ndi cações de cliente e simno ajustamento dos me mbros da rede às regras e normas que a regem, mantendo-se todos comprometidos comos objetiv os e int eresses da rede.

Po rtanto, a parti r da cooperação, passa a haver uma relação de soma positiva, emque todos ga nham e ninguém perde (AXELROD, 1984), ou seja, para sobreviveremno merc ado os consul tores se unemem red e atuando em busca do equilíbrio de Nash (Teoria dos Jogos).

\section{Permanência da re de e compr omet imento}

A confiança, o comportamento étic o e o comprometi mento são c ondi ci onant es para a cooperação, segundo as correlações ap resent adas no Apêndice B. Est es elementos, em conjunto, constituem base para que a rede se estabel eça com uma perspectiva de longo prazo, conservando ou melh orando a relação com as demandas do mercado segundo $89 \%$ dos consul to res.

A questão do comi ssionamento, acima referida, como exemplo típico da expect ativa de co mp ort amento éti co, volta rel acionada comas obrevivênci a da red e.

[Com a prática da corretagem] não se está preservando o int eresse do diente. Essa é uma sit uação fora de éti ca. Ela aumenta o custo para o diente. Ela [a rede] não se perpetua no mercado, porque [...] vai gerando a des confiança. Mat ou a razão de ser da rede. (Consultor 3, em entr evista coleti va, 23/11/2006)

O comprometimento entre os membros se dá, seg undo os consultores entrevistados, pelo compromisso de atender bem aos clientes indicados, trocar informaçõ es e experiências, e pela busca das indicações profissionais requeri das por cada um dos seus clientes. Est e comprometi mento não signi f ca, contudo, que cada membro deva devol ver in dic ações aos demais, o que indica uma rel ação assimétrica em termos de cont eúdo e de int ensidade de "troca" entre os me mbros. Nas palavras do Consultor 4:

Nesses dez anos eu nunca recebi uma indicação para mim, e nem por isso eu deixo de indicar para el es. Porque há consultores que estã o mais envolvidos no mercado [...] Quant o mais exp eri ência mais as pessoas os proaur am. [...] Esse pessoal, por exemplo, que eu indico não tem nenhuma obri gação comigo e eu nem quero nenhuma obrig ação. Nã o espero ter nenhum r etorno. (Consult or 4, entr evista col etiva,23/11/2006)

O importante é que cada membro busque não só o s eu próp rio interesse, mas os objetivos da rede: o mel hor para o cliente -,p ara que s e mantenha sua persp ectiva del ongo prazo.

Adi ciona-se a isto, de acordo com $89 \%$ dos consultores, a necessidade da expectativa de que os demais membros se comprometam dado que a expectativa de cada consultor ou empresa de cons ultoria em rel ação ao comprometimento dos demais, especi al mente por s er baseada em relação prévi a, exerce infuência sobre seu próprio comprometimento. Ess a suposição de Anderson e Weitz (1992) aparece nas correlações entre "comprometimento confr me compromet imento" e "relação prévi a" apresentadas no Apêndice B.

Anderson e Weit z (1992) fal am dos investi ment os de entrada e manut enção, ou a possibilidade de sua perda (STANLEY; MARKMAN, 1992), como fator significativo para manter o comprometimento. No caso das redes de consultoria organi zacional, como mostram $83 \%$ dos consultores, a perda não é só de investimento ou manuten ção, é mai or, refer e-s e à sobrevivência no mercado. Contudo, a perspectiva desse fi to não apa receu, no survey ou nos depoiment os, como algo qu e favo rece o comprometimento ordinário c oma rede.

No momento em que a gente faz parte de uma rede, a dissolução não é fácil. Não é fácil em termos de empresa e não é fácil em termos das pessoas que estão a ela vinculadas. Alguns poderiam estar 
precisando da rede para entrar no mercado, ficaria bem mais fácil. Existe também a questão da exigên cia das empr esas de vocêter uma vinculação a uma instituição, não é? Para que o contrato aconteça, então você teri a que estar vinaulado a uma org anização. E qua ndo há a dissolução há as implicações financeiras. (Consultor 1, entr evista coletiva,23/11/20 06)

As variáveis que assegur amo comprometiment o d os consultores e empres as de consultoria são: a dependên cia entre os parceiros, dec orr ent ed an ecessidade de co mplementaridade de competências ou de volume (corr el ação de 0,23); as regras e normas est abelecidas para regul ar as ações na rede (correlação de 0 25); e os benef cios, diretos e indiretos gerados pela participação na rede, conforme evi denciam as pal avras do Consultor 2: “[...] e continuar participando desta rede vai depender de quanto isto está me trazendo de beneficios. Eu não vou continuar [...] se ela não estiver agregando valor"( entre vista coletiva,23/11/2006).

A decisão de ent rada na rede pode ocorrer de forma consciente, com cálculo de custos e beneficios, ou de forma natural, em decorrência da sua dinâmica de atuação. Nesse momento, segundo os consultores entrevistados, não se considera a possi bilidade de desli gamento fituro da rede, muito meno s os prejuí zos disso result ant es ( $\mathrm{t}$ ais como perdas e transtornos). A s aíd a, por sua vez, se dá em decorrên cia do não atendimento das expectativas de al cance de interesses grup ais e indi viduais. Dest a forma, os consultores ou empresas de consultori a que não atuaremem busca dos interesses comuns à rede e dos seus próprios, sem possibili dade de exclusão de qualquer dos dois, tendema se desligar da rede.

\section{Algumas reflexões adicionais}

Ainda que o enfoque metodológico dessa pesquisa seja o descritivo, dada sua caracterí stica exploratória, as anál ises mais i mediatamente presas aos dados ensejamal gumas ref exões adicionais.

Por que as rel ações pess oais sensíveis - os padrões de comportamento ético, a inseparabilidade da di mensão pesso al à profis sional na composição da confiança, a exigência de amadureci mento prévio das relações $p$ ara ingress o na rede, o receio de que a formal ização das relações organizaci onais prevaleça sobre as p essoais, etc. s urgem co mo o traço mais caracteństico na formação e manutenção dess e tip o de re de soci al? Por que os int ere sses e as pressões de mercado contam menos? Po de-se, prelimi narmente, levantar a hipótes e de que aí se proj eta o campo sensível das próp rias relações consul tor-cli ent e. A consultori a de tipo organizaci onal - onde o consultor tem acesso a in frmações est ratégicas ou deli cadas, envol vendo sócios eplanos empre sariais, onde se tocam as mazelas e os valores típi cos da cultura organizacional, onde a solução de conflitos ou o difi cil esforço de mudança são o próprio objeto de seu trabalho - não é uma prestação de serviço s o rdi nária, bemco mo não t em o padrão co mum das relações comerciais, com evidência objetiva do resultado pelo qual se pag a. A 1 egiti midade do consul to $\mathrm{r} n$ ão é asseg urada l egalmente $n$ em mesmo por al guma convenção de mercado. Ela t emque s er conquistada pelo desempenho e é fácil de s er perdida. No contexto da consultori a organizacional não está ausente a di mensão terapêtica do próprio relaci onamento - base do serviço prestado pela clínica médica e psicológica.

Nesta pesquisa, estudou-se, parti cul armente, redes de consult ores ou de peque nas empresas de consultoria. Que levam seus result ados a pensar, consideradas as grandes empresas de consul toria, que, s emdú vida, constituem fenô men o de mercado muito mais forte e puj ant e? No caso dos consultores indi viduais e pequenas consultorias, a rede de confiança e compl ementaridade muda as rel ações de conc orrênci a. Inclusi ve, como s e viu, a chamada "pros pecção", por um consult or, d e novos clientes já assistidos no âmbito da própria rede, é eti cament e veda da. Nas grandes empresas de consultoria há cert aint egração vertical de divers os serviços ou etapas de projetos de mudança e reorienta ção de empresas. Entre elas, a con corrência é estratégica e funciona como p ara quaisquer empresas present es em um mes mo mercado. Eventuais parceri as não s e operamcomo nas redes descritas nessa pesquisa -, não passamde trocas de int eresse. Essa di ferença ressalta o caráter especial e único do fenômeno a qui estudado, e, enqu ant o o afast a de padrões típicos de mercado competitivo, o apro xima, descritivamente, de redes sociai s surgidas de afini dades ep or interesses cooperati vos di versos . 


\section{Conc lusão}

Investi gand o campo em que não se encontram pesqui sas específicas, as redes de consult oria organizacional, este estudo tentou inici al mente trazer elementos mais si gni f cativos da vast a liter atura sobre redes sociais. As características aí achadas na formação de redes foram apresentad as a uma amostra de 90 consultores de pequenas e mé dias consultori as. Os result ados numéricos mais expressivos desse survey foramco mentados em longa entr evi stac oletiva compequeno grupo de consultores mais exp ei entes.

$\mathrm{Na}$ formação das redes de consultoria organizacional, quat ro elementos relacion ais se apresentam como altament ei mport antes: i) expect ativa de cooperação entre os membros; ii) comprome timento deles com a rede - às vezes formalizada e com marca própria - e entre si; iii) confiança pessoal em outros cons ultores e na institui ção associada (empresa de consul toria); e iv) expect ativa de comportamentos éticos. Os dois últimos encontram se no âmago do fenômeno por constit uírem não só el ementos ess enci ais como também base para os demais.

Para garantir os quatro el ement os essenciais à formação das redes, três mecanismos têm sido utilizados: i) estabel eci mento de relação prévia entre os membros como fonte de informação para avaliação da decisão de conf ar-lhes a in dic ação, o s erviç o ou projeto; ii ) defini ção, 6 rmal ou tá cita, de regras e nor mas que re gule mas prátic as e ações dentro da rede, g arantindo a manutenção dos quatro elementos; iii) limitação do tamanho da rede pela capacidade de manutenção dos quatro elementos essenciais, possibilitando a ge ração de beneficios para a rede, s eus membro s e o mercado.

Conclusão igualmente import ante, e alt amente signi ficativa para ent endi mento da dinâmica das redes de consultori a organi zacional, é que, nes se mercado de comerciali zação de um bem intangível, em que os conhecimentos esp ecíf cos dominados por cada consultor constituem fator principal, os consultores têm sua i mport ância individual ressaltada. Emdec orrência, há uma personi ficação da consultoria no consultor, gerando cert a prevalênci a das relações pes soais sobre as organi zacion ais, mesmo dent ro de uma rede da qual empresas fazemparte.

Exploratóri a e descritiva, a pesquisa mostrou o potencial de especificidade das redes de consultores e pequenas consultorias, sugerindo com is so ser campo para confrontos mais arrojados com o conhecimento hoje abundante sobre redes sociais e para estudos comparati vos tanto comou tros ti pos de rede social que se $6 \mathrm{rmam}$ por afinidades e int eresses cooperativos, quant o emout ro extremo comas grandes empre sas de co nsul tori a, que funci onamestritamente d entro de padrões rel acionais de merc ado. 


\section{Referências}

ALMEIDA, Nuno M. C.; COELHO, Arnaldo; COEHO, Filipe. Determinantes do Compromisso nas Relações Empresar ia ś. In: Encontro da Associação Nacional de Programas de Pós-Graduação em Administração - ENANPAD, Anais..., Porto Alegre: ANPAD, 2004, 1 CD-ROM.

ANDERSON, E; WETZ, B. The us e ofpleadge s to bu ild and su stain co mmitment in distr ibution channels. Journal of Market ing Reser ach, Feb.,p.18-34, 1992

AXELROD, R A e volução da cooperação. São Paulo, 1984.

BACHMANN, Reinhard. Trust, Power and Contro lin Trans-Organization al Relations. Organ ization Studies, v. 22, n. 2, p. 337-35 7, mar.2001.

BALESTRO, Mo is és, V. Confiança em rede:a exper iência da rede de e stofador es do pób mov eleiro de Bento Gonçalves. 2002. Dissertação (Mestrado em Administração )- Univers dade Federal do Rio Gran de do Sul, Porto Alegre, 2002

B ARNES, J. A. Ca ss and committ ees ni a Norweg àn is and par ish. Human Relat ions, v. 7,n. 1, p.39-58.1954.

CAMPEL ODESOUZA, B. A T eoria da mediação cogn it iva:os impactos cogn itivos da hipercultura e da mediação dig $t$ al.Tese (Doutorado) - Programa de Pós-Graduação em Psicolog ia ,D epartamento de P sicologia, Universidade Federalde Pernambuco, Recife, 2004.

CÂNDIDO, G.A.; DE ABREU, A F.F atore scr $t$ icos de sucesso no processo de for mação, de sen volvimento e viabilização de redes organizac ónais: um estudo explor atório. In: Encontro da Assoc iação Nacional de Programas de Pós-Graduação em Admin istr ação - ENANPAD, Ana í ..., Curitiba: ANPA D, 2004. 1 CD ROM.

CARDOSO, V.C.; ALVAREZ, R. R; CAULLIRAUX, H. M. Gestão de competências em redes de organizações: discu ssões t eór icas e metodológcias acerca da problemática envolvida em projetos de implant ação. In: Encontro da Associação Nac b́nal de Programa s de Pós -G raduação em Admin śtração - ENANP AD, Ana is ..., Sa lvador: ANPAD, 2002, 1 CD-ROM.

CARVALHO, M. R O. Redes soc iais: convergências e paradoxos na ação estratégica. In: Encon tro da Ass ociação Nacional de Programa sde Pós -Graduação em Admin stração - ENA NPAD, Anais..., Salvador :ANP AD,2 002. 1 CD-ROM.

CASTEUS, Manuel. A socied ade em rede - A era da informa ção: economia, sociedade e cu tura. S ão Pau lo: Paz e Terra, 1999, v.1.

COASE, R. H The Nature of the firm. Econômic a,nov. 1937.

COOPER, D.R.; SCHINDLER, P.S.Métodos de Pesquis a em Admin istr ação.7.ed. Po rto Akgre:Bookman,2003.

DONADONE, J. C. “Os hunos já chegaram!': dinâmic a organizacional, difusão de conceitos gerenciais e atuação das con sultor ias. Tese Doutorado em Engenhar ia de Produção) - Escola Politécn ica, Universidade de S ão Pau b, São Pau lo, 2001.

O mercado int ernacional de consultorias nas últimas déc adas: crescimento, diver sificação e formas de disputa. Ca derno de Pesquisa em Adminis tr ação,São Pa u lo, v. 10,n. 2,p. 1-15, abr/jun. 2003.

GRANOVET TER, Mar k. The str ength ofwe ak t ies. Ame ric an Journalof Soc iology,78:1 360-80, 1973. The strength of weak ties:a network theory revisited. In:Sociologic alTheory. San Fr anc isco, 1983, p.201-233.

.Economic Action and Social Structur e: The Problem of Embededness. America n Journal of Soc iology, v. 91, n. 3, p. 481-510, Nov. 1985 .

GULATI, Ranjay. Social structure and allianc e formation patterns: a long tudinal analysis. Administrative Science Quarterly, Ann Harbor, v. 40, p. 619-652, 1995.

INOJOSA, Ro se Marie. Redes de Compromisso. Rev í ta de Administração Pública, RAP. Rio de Jan eiro: FGV,33(5), p.1 15 - 14 1, set / out .1999 . 
KRAMER, R.M. Trust and distrust in organizations:e merg ing perspectives, endur ing questions. Annual Review of Ps ychology, v. 50 ,p. 569-98, 1999 .

LANE, Christel; BACHMANN, Reichard. The social constitution of trust: supplier relat bns in Brita in and Ger many. Organizat ion Studies, v. 17, n. 3,p. 365 - 395, summer 1996.

L IMA, G. A. N.; LIMA, J. B.; TAKAKI, H. R. C. Confiança e Apr end żagem Organżaciona Ina F ormação de Consórcios de PMEs para Exportação. In: Encontro da As soc iação Na cional de Progra mas de Pós-Graduação em Ad min istração - ENA NPAD, Anais..., Porto Alegr e: ANPAD, 2004,1 CD-ROM

LOPES, Fernando; BADI, Mariana. Laços sociais e for mação de arranjos organizaciona ś cooperativos: proposç̧ão de um modelode análise.Rev ista d e Admin is tração Con te mporânea, Rio de Jan eiro, v. 9, n.2, p. 81-101, abr/jun., 2005.

L UHMANN, Niklas. Confianza. Rubi (Barcelona): Anthro pos Ed tor à l, 2005.179 p.

MATTOS, P. L. C. L. A entrevista não-estruturada co mo forma d e conv ers ação: razões e sugestõ es para sua aná lise. Rev í ta de Ad min is traç ão Pública, RAP.p. $823-84$ 7, JuVA go, 2005 a.

. Consultoria organżacional fora do grande circuito. Relatório parcial de projeto de pesquisa - CNPQ. Re cife: UFPE, 2005b. [Docu mento não pub licado]

MIZRUCHI, Mark S. Análise de redes socia is: avanços r ecente se controvérs à s atuais. Revis ta de Admin istraç ão de Empres as, v. 46, n. 3, p.72-86, jul/ set., 2006.

MORE NO, J. L. Who sha I sur vive? Wash ington, DC: Nervous and MentaID iseas e Publish ing Co, 1934.

MORGAN, R. M; HUNT, S. The Co mmitment-Trust Theory o fre lation ship market ing. Journalof Marketing, Ju ע, p.20-38, 1994.

PASSUELLO, Caroline B. Confiança e Risco em Decisões Es tratégicas: uma análise a part ir de elementos do sistema exper ienc ial. Dí sertação (Mestrado emAdministração) - UNISINOS,São Leopoldo,2006.

PASSUELLO, Caroline B.; SWIRSKIDE SOUZ A, Yed a. Confiança e risco em processo s decisórios. In: Encontro da As soc a ção Na cional de Programas de Pós -Graduação em Adm in istraç ão - ENANPAD, Anais..., Bra sília : ANPAD, 20 05. 1 CD-ROM.

PEIXOTO, A. Circulação de saber nas redes sociais inf ormais em o rganizações privad as: condicionant es e impactos. Dissertação (Mestrado) - Programa de Pós-Graduaç ão em Admin istração, Departamento de Ps čologia, Univer sidade Fed era Ide Pern ambuco, Recife, 2006.

PUTNAM, Robert D. Comunida de e democr acia: a experiênc à da It ália modema. Rio de Janeiro: Fun dação Getúlio Vargas, 1996. $260 \mathrm{p}$

RENNÓ, Lucio R Confiança interpessoal e comportamento polt ico: microfundamentos da teor ia do capita I socialn a A mérica Latina. Opin. Publica. Campinas, v. 7, n. 1, 2001. Disponível em : $<$ htt p:// wwwscielo.br/scielo.ph p? script=sci_arttext\&pid=S0 1046276 20010-00100003\&lng=en \&nrm=iso>. Acesso em: 08 a go. 2006 .

SÁ, Márcio; ABELSON, Sérgio. Redes Sociais, pessoais e de Negócios. Trabalho apresent ado na disciplina de Marketing E stratégico do Programa de Pós graduaç ão em Admin istração - PROPAD, Un iver sidade Federa Ide Pern ambuco, Recife, 20 04.

S IMMEl G. Conf lict \& the we bof group- affiliat ions. New York: The Fr ee Pres s, 1969.

STANEY, Scott C.;MARKMAN, Howard J. Assessing commitment in personal re lation sh ip.J our nal of Marriage and the Family, v. 54,p. 595608 , Aug. 1992.

WELLMAN, B. St udy ning Personal Commun it ies. In: MARSDEN, P.; L IN, N (ed.). Social Structu re and ne twor k an alys is . Bev erly Hills: Sage Public ations, 1982.p. 61-80.

Structura lanalysis: From method and metaphor to theor y and subst ance. In: WEШMAN, B; BERKOWIZ, S. D. Social struct ures:a network approach. New York: Cambridge Univer sity Press, 1988. p.1961 
WIШIAMSON, O. E.Mar kets and Hier archies: Analysis and Antitru st Implication s. Ne wY ork :Free Pres s, 1975.

WOOD, Thomaz Jr. DE PAULA, Ana Paula P. Empre sas de consultoria no Brasil:um estudo múlt iplo de casos. EAESP/FGV/NPP:

Rela tór io de Pesquisa, 2004. 


\section{Apêndices}

Apêndice A-Avaliação dos fat ores rel acionais na formação das r edes deconsultoria or ganizaci onal

Tabela 1: Fatores relacionais na formação das redes de consultoria organizacional

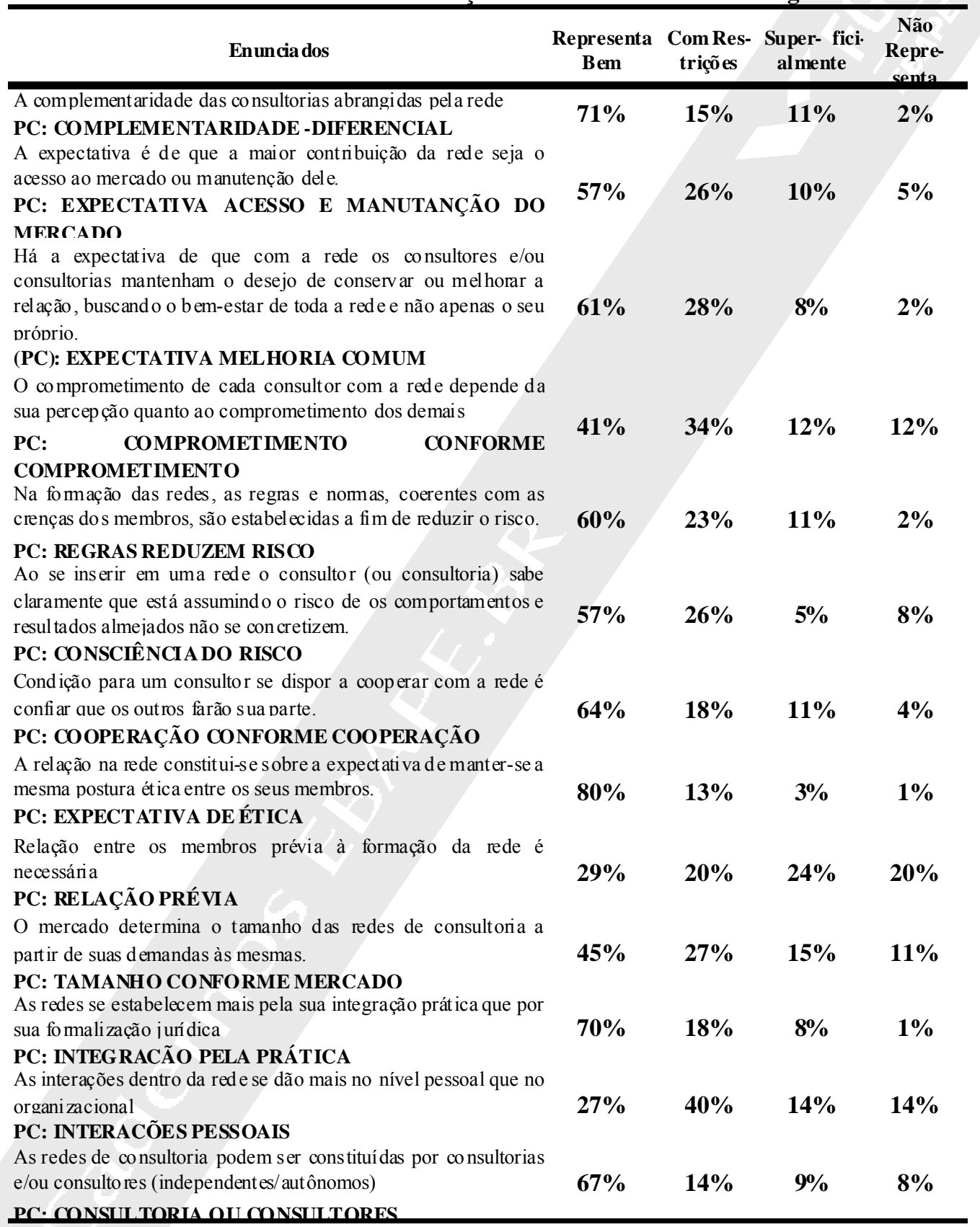


Apêndice B - Cor relą̧ões dos fatores relacionais na formação das redes de consultoria organi zacional

\begin{tabular}{|c|c|c|c|c|c|c|c|c|c|c|c|c|c|}
\hline & $\begin{array}{l}\text { expectativa } \\
\text { melho ia } \\
\text { comum }\end{array}$ & $\begin{array}{c}\text { comprometimento } \\
\text { conforme } \\
\text { comprometiment }\end{array}$ & $\begin{array}{l}\text { regras } \\
\text { reduzem } \\
\text { risco }\end{array}$ & $\begin{array}{l}\text { cons ciênc ia } \\
\text { dorisco }\end{array}$ & $\begin{array}{c}\text { co ope raçẵo } \\
\text { co nfo me } \\
\text { co ope ração }\end{array}$ & $\begin{array}{l}\text { expe ctativa } \\
\text { de ética }\end{array}$ & $\begin{array}{l}\text { relaç ão } \\
\text { prévia }\end{array}$ & $\begin{array}{l}\text { integracãao } \\
\text { pe lap rática }\end{array}$ & $\begin{array}{l}\text { nter ações } \\
\text { pe sso ais }\end{array}$ & $\begin{array}{c}\text { consult onia } \\
\text { e } \\
\text { consultores }\end{array}$ & $\begin{array}{c}\text { Complemen- } \\
\text { tainade } \\
\text { dife re ncial }\end{array}$ & $\begin{array}{c}\text { Expe c.acesso } \\
\text { e manut.do } \\
\text { merc ado }\end{array}$ & $\begin{array}{l}\text { Tamanho } \\
\text { conforme } \\
\text { mercado }\end{array}$ \\
\hline $\begin{array}{l}\text { ex pecc tativa melho nia } \\
\text { comum }\end{array}$ & 1,00 & 0,15 & 0,25 & $-0,05$ & 0,23 & 0,39 & 0,04 & 0,28 & $-0,11$ & 0,13 & 0,23 & 0,11 & 0,22 \\
\hline conforme & & & & & & & & & & & & & \\
\hline $\begin{array}{l}\text { co mp ro me timento } \\
\text { regr as red uzem }\end{array}$ & 0,15 & 1,00 & 0,23 & 0,28 & 0,32 & 0,13 & 0,28 & 0,21 & 0,05 & 0,06 & 0,17 & 0,04 & 0,07 \\
\hline ríco & 0,25 & 0,23 & 1,00 & 0,04 & 0,26 & 0,28 & $-0,06$ & 0,26 & $-0,04$ & 0,01 & 0,10 & 0,02 & 0,18 \\
\hline $\begin{array}{l}\text { consciê ncia do nisco } \\
\text { co oper raçấo }\end{array}$ & $-0,05$ & 0,28 & 0,04 & 1,00 & 0,22 & 0,25 & 0,29 & 0,19 & 0,15 & $-0,06$ & 0,04 & 0,00 & 0,12 \\
\hline $\begin{array}{l}\text { confo me } \\
\text { cooperaçẫo }\end{array}$ & 0,23 & 0,32 & 0,26 & 0,22 & 1,00 & 0,26 & 0,00 & 0,47 & $-0,09$ & 0,13 & 0,27 & 0,05 & 0,38 \\
\hline ex pec tativa de ética & 0,39 & 0,13 & 0,28 & 0,25 & 0,26 & 1,00 & 0,09 & 0,29 & 0,10 & 0,03 & 0,08 & 0,02 & 0,15 \\
\hline $\begin{array}{l}\text { relação p révia } \\
\text { int egr acã̃op ela }\end{array}$ & 0,04 & 0,28 & $-0,06$ & 0,29 & 0,00 & 0,09 & 1,00 & 0,05 & 0,08 & 0,16 & 0,04 & 0,26 & 0,14 \\
\hline prát c a & 0,28 & 0,21 & 0,26 & 0,19 & 0,47 & 0,29 & 0,05 & 1,00 & 0,05 & 0,23 & 0,41 & 0,11 & 0,30 \\
\hline int era cões s pesso ais & -0.11 & 0.05 & $-0,04$ & 0.15 & -0.09 & 0.10 & 0.08 & 0.05 & 1,00 & 0.05 & 0.04 & 0,08 & $-0,17$ \\
\hline $\begin{array}{l}\text { consultor es } \\
\text { Complement and ade. }\end{array}$ & 0,13 & 0,06 & 0,01 & 0,14 & 0,13 & 0,03 & 0,16 & 0,23 & 0,05 & 1,00 & 0,16 & 0,27 & 0,13 \\
\hline $\begin{array}{l}\text { dif ere ncial } \\
\text { Exp ec.a cess oe }\end{array}$ & 0,23 & 0,17 & 0,10 & 0,04 & 0,27 & 0,08 & 0,04 & 0,41 & 0,04 & 0,16 & 1,00 & 0,25 & 0,19 \\
\hline $\begin{array}{l}\text { man ut.do o mer cad o } \\
\text { Tam an ho con for me }\end{array}$ & 0,11 & 0,04 & 0,02 & 0,00 & 0,05 & 0,02 & 0,26 & 0,11 & 0,08 & 0,27 & 0,25 & 1,00 & 0,38 \\
\hline mercado & 0,22 & 0,07 & 0,18 & 0,12 & 0,38 & 0,15 & 0,14 & 0,30 & $-0,17$ & 0,13 & 0,19 & 0,38 & 1,00 \\
\hline
\end{tabular}

\footnotetext{
O site de busca nwwscholar.aoogle.combr ou www.google.combr não regis tra qualquer entra da para "redes de consult ria organizacional" ou "redes de consultores organizacionais". A busca co ntinua influ tífera mesmo com as en tradas em inglês ("netwo lks of organizational consultancy" e "network of organizational consultan ts", nesse último cas o have ndo brevśsima referência a uma organização nos Paises Baixos, cha mada UR University, fo mada por consultores ).

2 Pa ra presenvarsuas identidades, os quatio e ntievistados serão denominados "Consultor 1", "Co nsu ltor 2", "Consultor 3" e "C onsultor 4".
} 